\title{
Papers
}

\section{Psychological stress and cardiovascular disease: empirical demonstration of bias in a prospective observational study of Scottish men}

\author{
John Macleod, George Davey Smith, Pauline Heslop, Chris Metcalfe, Douglas Carroll, Carole Hart
}

\begin{abstract}
Objectives To examine the association between self perceived psychological stress and cardiovascular disease in a population where stress was not associated with social disadvantage.

Design Prospective observational study with follow up of 21 years and repeat screening of half the cohort 5 years from baseline. Measures included perceived psychological stress, coronary risk factors, self reported angina, and ischaemia detected by electrocardiography.

Setting 27 workplaces in Scotland.

Participants 5606 men (mean age 48 years) at first screening and 2623 men at second screening with complete data on all measures

Main outcome measures Prevalence of angina and ischaemia at baseline, odds ratio for incident angina and ischaemia at second screening, rate ratios for cause specific hospital admission, and hazard ratios for cause specific mortality.

Results Both prevalence and incidence of angina increased with increasing perceived stress (fully adjusted odds ratio for incident angina, high versus low stress $2.66,95 \%$ confidence interval 1.61 to $4.41 ; \mathrm{P}$ for trend $<0.001)$. Prevalence and incidence of ischaemia showed weak trends in the opposite direction. High stress was associated with a higher rate of admissions to hospital generally and for admissions related to cardiovascular disease and psychiatric disorders (fully adjusted rate ratios for any general hospital admission 1.13, 1.01 to 1.27, cardiovascular disease $1.20,1.00$ to 1.45 , and psychiatric disorders $2.34,1.41$ to 3.91$)$. High stress was not associated with increased admission for coronary heart disease (1.00, 0.76-1.32) and showed an inverse relation with all cause mortality, mortality from cardiovascular disease, and mortality from coronary heart disease, that was attenuated by adjustment for occupational class (fully adjusted hazard ratio for all cause mortality $0.94,0.81$ to 1.11 , cardiovascular mortality $0.91,0.78$ to 1.06 , and mortality from coronary heart disease $0.98,0.75$ to 1.27$)$.
\end{abstract}

Conclusions The relation between higher stress, angina, and some categories of hospital admissions probably resulted from the tendency of participants reporting higher stress to also report more symptoms.
The lack of a corresponding relation with objective indices of heart disease suggests that these symptoms did not reflect physical disease. The data suggest that associations between psychosocial measures and disease outcomes reported from some other studies may be spurious.

\section{Introduction}

Psychosocial factors-for example, psychological stress, are widely believed to be important determinants of heart disease. ${ }^{1-3}$ Exposures to such factors may influence health directly through neuroendocrine mechanisms or indirectly through their association with unhealthy behaviour. ${ }^{45}$

Much of the evidence supporting this hypothesis comes from studies relating self reported psychosocial measures, such as perceived stress, to self reported health outcomes. Individuals with a general tendency towards negative perceptions of different aspects of life may over-report both psychosocial adversity and symptoms of disease, leading to a spurious association between adverse psychosocial exposure and health. ${ }^{6}$

Studies using more objective outcomes have largely been conducted in populations where psychosocial adversity was associated with general social disadvantage. Therefore it is impossible to discount the possibility that the apparent "effects" of psychosocial exposure are due to residual confounding by other correlates of relative deprivation. ${ }^{7}$

An association between stress and social disadvantage is not inevitable, as the social distribution of perceived stress seems to vary with place and historical period. ${ }^{8}$ We explored the relation between self perceived stress and several indices of cardiovascular health within a workplace derived population of men in whom reported stress was not greater among the socially disadvantaged. ${ }^{9}$ By comparing the association between stress and a series of health outcomes dependent on self report to a different degree, we were able to assess the influence of reporting tendency.

\section{Methods}

Our investigation is based on a cohort of men recruited from 27 workplaces in Scotland between 1970 and 1973. Measurements at recruitment included
Department of
Primary Care and
General Practice,
University of
Birmingham,
Birmingham
B15 2TT
John Macleod
clinical research fellow
Department of
Social Medicine,
University of
Bristol, Canynge
Hall, Bristol
BS8 2PR
George Davey
Smith
professor of clinical
epidemiology
Pauline Heslop
research assistant
Chris Metcalfe
research assistant
School of Sport and
Exercise Science,
University of
Birmingham,
Birmingham
B15 2TT
Douglas Carroll
professor of applied
psychology
Department of
Public Health,
University of
Glasgow, Glasgow
G12 8RZ
Carole Hart
research fellow
Correspondence to:
J Macleod
j.a.macleod@
bham.ac.uk
bmj.com 2002;324:1247 
cardiovascular risk factors, "Rose" angina, and six lead resting electrocardiography. ${ }^{10}$ Perceived stress was measured with the Reeder stress inventory (box), a four item questionnaire instrument widely used during the 1960s and 1970s..$^{911-14}$

The same workplaces were revisited in 1977, a mean of 5 years and 2 months from first screening. Around $50 \%$ of participants were rescreened using the methods as in the initial screening. Our study is based on 5606 men at first screening and 2623 at second screening with complete data on all measures. Full descriptions of the methods and procedures have been published elsewhere. ${ }^{915}$

Men who died over the 21 years of follow up were identified through flagging at the NHS Central Registry in Edinburgh, which provides death certificates coded according to the ICD-9 (international classification of diseases, 9th revision). Deaths from cardiovascular disease were those covered by codes 390-459; coronary heart disease was covered by codes 410-414.

Data on hospital admissions for the same period were provided through linkage to the Scottish Morbidity Register. This has data on all admissions to Scottish hospitals, coded according to the contemporaneous

\section{Reeder stress inventory}

The Reeder stress inventory consists of four statements:

In general I am usually tense or nervous

There is a great amount of nervous strain connected with my daily activities

At the end of the day I am completely exhausted mentally and physically

My daily activities are extremely trying and stressful Participants indicate the extent to which each statement applies to them using a four point Likert format. Psychometric analysis of the responses of the current cohort indicated acceptable internal consistency (Cronbach's $\alpha=0.77$ ). Principal components analysis and maximum likelihood factor analysis yielded a one factor solution. A scoring system was employed to derive a summary score ranging from 1 (low perceived stress) to 8 (high perceived stress) as below.

0 , no response to one or more statement (that is, missing responses). These were omitted from the analyses

1 , "not at all" on all four statements

2 , not at all on any three with any other response on the fourth

3 , not at all on any two with "not very accurately" on the other two

4 , not at all on any one or two with any other response on the remainder but not those in 3

5 , all others not specified on 1, 2, 3, 4,6, 7, or 8

6 , "to some extent" on all four or to some extent on three and "exactly" on the fourth

7 , exactly on any three with to some extent or "not very accurately" on the fourth or exactly on two with to some extent on two

8 , exactly response to all four statements.

This system was devised by Reeder et al. ${ }^{13}$ Stress scores derived using a more conventional "Likert" approach to scoring (that is, attaching a score of 1-4 to each response category and summing these across the four items) showed the same pattern of relations with all outcomes and covariates. revision of the international classification of diseases. ${ }^{16}$ We considered admissions to general hospitals (SMR1) and to psychiatric hospitals (SMR4). We used codes appearing in any diagnostic position from the final consultant episode. Cardiovascular disease and coronary heart disease were coded as above. We considered acute myocardial infarction (code 410) and angina (413) separately. We considered as a separate group hypertension, ill defined heart disease, varicose veins, and haemorrhoids, hypotension, and "other" circulatory disorders (401, 429, 454-459), and psychiatric disorders (295-298, 300, 303-309, 311, 312, and 316).

Stress scores (1-8) were categorised as high (6-8), medium (4 and 5), or low (1-3; box). We calculated the distribution of coronary risk factors and the prevalence of angina and ischaemia at first screening associated with each category. We standardised all estimates for age and occupational class. We used linear regression to test for linear trends across stress categories.

We calculated the odds ratios through logistic regression for incident angina and ischaemia at second screening according to reported stress category at initial screening. We defined "incident" angina or ischaemia as angina or ischaemia at second screening in a participant without angina or ischaemia at first screening. We included in these models age, occupational class, screening interval, and cardiovascular risk factors. We calculated odds ratios through logistic regression models adjusted for age for incident angina and ischaemia associated with established cardiovascular risk factors.

We evaluated the relation between the different categories of hospital admission and different levels of perceived stress and risk factors as a rate ratio using negative binomial regression. This elaboration of Poisson regression accommodates variation in the propensity for admission to hospital across individuals. ${ }^{17} \mathrm{We}$ considered admissions over a period of 21 years from first screening. We included age, occupational class, and cardiovascular risk factors in models.

We calculated categories of mortality associated with angina, ischaemia, established cardiovascular risk factors, and perceived stress in proportional hazard models including age. We took survival time to start at first screening. We added occupational class and coronary risk factors sequentially.

We repeated the prospective analyses to test for a trend across the three categories of stress. We undertook all analyses with STATA 6.0 (Statacorp, College Station, TX, USA).

\section{Results}

Perceived stress showed a graded association with occupational class from a mean stress score of 4.4 in social class I to a mean stress score of 2.8 in social class $\mathrm{V}$ ( $\mathrm{P}$ for trend $<0.001)$. Table 1 shows the distribution of cardiovascular risk factors and prevalence of angina and ischaemia by perceived stress at first screening. Higher stress is associated with an adverse pattern of behavioural risk factors but shows no clear relation with physiological risk factors. A substantial trend of higher prevalence of angina with higher stress is apparent. Conversely, there is a weak, inverse association of prevalent ischaemia with baseline stress. These cross sectional associations were the same when 
Table 1 Relations between perceived stress, coronary risk factors, and prevalence of angina and ischaemia at first screening. Values are numbers (percentages) unless stated otherwise

\begin{tabular}{|c|c|c|c|c|}
\hline & \multicolumn{3}{|c|}{ Stress } & \multirow[b]{2}{*}{$P$ for trenc } \\
\hline & Low $(n=1826)$ & Medium $(\mathrm{n}=3037)$ & High $(n=743)$ & \\
\hline Smoking $>20$ cigarettes daily & $337(18.5)$ & $651(21.3)$ & $196(26.5)$ & $<0.001$ \\
\hline Consuming $>15$ units of alcohol weekly & $554(27.2)$ & $831(29.2)$ & $223(29.5)$ & 0.031 \\
\hline Taking $<3$ hours exercise weekly & $391(19.4)$ & $553(18.8)$ & $194(26.4)$ & 0.004 \\
\hline Body mass index $>25 \mathrm{~kg} / \mathrm{m}^{2}$ & $976(52.7)$ & $1536(49.9)$ & $352(46.7)$ & 0.004 \\
\hline Diastolic blood pressure $90 \mathrm{~mm} \mathrm{Hg}$ & $502(25.9)$ & $737(24.8)$ & $175(23.3)$ & 0.12 \\
\hline Plasma cholesterol concentration $>5.5 \mathrm{mmol} / \mathrm{l}$ & $1073(60.3)$ & $1944(62.5)$ & $465(60.3)$ & 0.92 \\
\hline $\mathrm{FEV}_{1}<90 \%$ of predicted value & $729(36.7)$ & $1107(37.8)$ & $277(35.6)$ & 0.69 \\
\hline Baseline prevalence of angina (\%) & $79(3.8)$ & $196(6.8)$ & $70(8.7)$ & $<0.001$ \\
\hline Baseline prevalence of ischaemia (\%) & $118(6.2)$ & $169(5.7)$ & $36(4.7)$ & 0.11 \\
\hline
\end{tabular}

Standardisation by the direct method for age and occupational class.

$\mathrm{FEV}_{1}=$ forced expiratory volume in one second.

analysis was restricted to members of the cohort screened twice (data not shown).

Table 2 shows the relation between established cardiovascular risk factors at baseline and subsequent events. In general, established risk factors predicted increased events. Smoking was not related to incident ischaemia, possibly because of a heightened risk of death in smokers with myocardial ischaemia, who were therefore less likely to be alive at the time of second screening. Associations were slightly weaker in relation to "Rose" angina and admissions for angina.

Table 3 shows an age adjusted odds ratio for development of incident angina of 2.32 in participants with high stress compared with those with low stress. Further adjustment for social class and cardiovascular risk factors strengthened this relation. A dose-response association between stress and incident angina was seen. There was, however, an apparent weakly inverse relation between high stress and incident ischaemia.

Table 4 shows a higher rate of hospital admission with higher stress. This was most strongly apparent in relation to psychiatric disorders. Higher stress also strongly predicted admissions related to hypertension, varicose veins, and ill defined cardiovascular conditions. Patients admitted with angina showed a weak positive association with stress. Those admitted for acute myocardial infarction showed a weak inverse association with stress. Adjustment for social position and risk factors made little difference to most of these estimates. Adjustment for cardiovascular risk factors made little difference to the relation between stress and admissions for psychiatric conditions, and confining analysis to only the principal discharge diagnosis did not alter any patterns (data not shown).

Table 5 shows a moderate inverse relation between stress and mortality from all causes, cardiovascular disease, and coronary heart disease. Adjustment for occupational class substantially attenuated this association, with further adjustment for risk factors having little influence. Perceived stress was relatively stable among those men screened twice (weighted $\mathrm{k}=0.45$ ). In these men both mean stress score and change in stress score showed essentially the same relation with mortalitythat is, higher mean stress and increased stress were associated with lower mortality (data not shown).

\section{Discussion}

A strong and substantial relation exists between self reported stress and self reported symptoms of coronary heart disease. A relation of similar strength and magnitude is seen between stress and admission to hospital for psychiatric disorders. A weaker and less substantial relation is apparent between stress and admissions to a general hospital, including those broadly classified as related to cardiovascular disease.

Table 2 Established cardiovascular risk factors at first screening and subsequent risk of coronary events (incident angina and ischaemia at five years, admissions for coronary heart disease, admissions for acute myocardial infarction, admissions for angina over 21 years, mortality from coronary heart disease at 21 years, all adjusted for age)

\begin{tabular}{|c|c|c|c|c|c|c|}
\hline & \multicolumn{2}{|c|}{ Odds ratio $(95 \% \mathrm{Cl})$} & \multicolumn{4}{|c|}{ Hazard ratio $(95 \% \mathrm{Cl})$} \\
\hline & Incident angina & Incident ischaemia & $\begin{array}{l}\text { Admissions for coronary } \\
\text { heart disease }\end{array}$ & $\begin{array}{l}\text { Admissions for acute } \\
\text { myocardial infarction }\end{array}$ & $\begin{array}{l}\text { Admissions for } \\
\text { angina }\end{array}$ & $\begin{array}{l}\text { Mortality from coronary } \\
\text { heart disease }\end{array}$ \\
\hline $\begin{array}{l}\text { Smoking (current } \\
\text { smokers } v \text { others) * }\end{array}$ & 1.50 (1.06 to 2.12 ) & 0.99 (0.71 to 1.37) & 1.50 (1.26 to 1.78$)$ & 1.58 (1.32 to 1.89$)$ & 1.18 (0.84 to 1.67$)$ & 1.75 (1.48 to 2.07 ) \\
\hline $\begin{array}{l}\text { Plasma cholesterol } \\
\text { concentration } \\
(\mathrm{mmol} / \mathrm{l}) \dagger\end{array}$ & $1.06(0.90$ to 1.25$)$ & 1.35 (1.16 to 1.50$)$ & 1.26 (1.16 to 1.37$)$ & 1.31 (1.20 to 1.42$)$ & 1.25 (1.05 to 1.49$)$ & $1.30(1.20$ to 1.39$)$ \\
\hline $\begin{array}{l}\text { Diastolic blood pressure } \\
\quad(\mathrm{mm} \mathrm{Hg}) \ddagger\end{array}$ & $1.13(0.96$ to 1.32$)$ & 1.24 (1.07 to 1.45$)$ & $1.12(1.03$ to 1.20$)$ & 1.18 (1.09 to 1.28$)$ & 1.16 (1.00 to 1.34$)$ & 1.36 (1.28 to 1.46$)$ \\
\hline Angina§ & & & 2.31 (1.69 to 3.17 ) & 1.83 (1.35 to 2.49$)$ & 2.89 (1.61 to 5.22$)$ & 2.20 (1.72 to 2.80$)$ \\
\hline Ischaemia§ & & & 1.41 (1.00 to 1.98$)$ & 1.31 (0.93 to 1.84$)$ & 0.68 (0.31 to 1.51$)$ & 3.02 (2.41 to 3.78$)$ \\
\hline
\end{tabular}

All mortality analyses are on 5606 men with complete data on all measures, analyses for incident angina are on 2487 men with no angina at first screening and who were screened twice, analyses for incident ischaemia are on 2501 men with not ischaemia at first screening and who were screened twice.

${ }^{\star}$ There were 3140 current smokers at first screening, 1310 current smokers did not have angina at first screening and were screened twice, 1350 current smokers did not have ischaemia at first screening and were screened twice.

tOdds ratios and hazard ratios are those associated with $1 \mathrm{mmol} / \mathrm{l}$ increase in plasma cholesterol concentration.

¥0dds ratios and hazard ratios are those associated with $10 \mathrm{~mm} \mathrm{Hg}$ increase in diastolic blood pressure.

$\S 345$ men had angina at first screening. Angina was considered present if pain or discomfort over sternum or left chest and arm while hurrying or walking uphill caused participant to slow down or stop, pain subsiding in 10 minutes or less. 323 men had ischaemia at first screening. ECGs were coded according to the Minnesota system. Ischaemia was encompassed by codes 1.1-1.3, 4.1-4.4, 5.1-5.3, and 7.1. 61 men had both angina and ischaemia. 
Table 3 Odds ratio (95\% confidence intervals) for incident angina and ischaemia at second screening by reported stress category at first screening (low stress as baseline, mean follow up period 5 years 2 months)

\begin{tabular}{lccc}
$\begin{array}{l}\text { Perceived stress } \\
\text { Incident anginat: }\end{array}$ & $\begin{array}{c}\text { No of } \\
\text { participants }\end{array}$ & $\begin{array}{c}\text { Adjusted for age } \\
\text { only }\end{array}$ & $\begin{array}{c}\text { Adjusted for age, social class, } \\
\text { screening interval, and risk } \\
\text { factors* }\end{array}$ \\
\hline High stress & 312 & $2.32(1.43$ to 3.78$)$ & $2.66(1.61$ to 4.41$)$ \\
\hline Medium stress & 1338 & $1.22(0.82$ to 1.82$)$ & $1.37(0.91$ to 2.08$)$ \\
\hline Low stress & 837 & 1.00 & 1.00 \\
\hline P for trend & \multicolumn{4}{c}{0.002} & $<0.001$ \\
\hline Incident ischaemiał: & 337 & $0.63(0.34$ to 1.15$)$ & $0.67(0.36$ to 1.26$)$ \\
\hline High stress & 1349 & $1.04(0.73$ to 1.48$)$ & $1.03(0.71$ to 1.49$)$ \\
\hline Medium stress & 815 & 1.00 & 1.00 \\
\hline Low stress & & 0.27 & 0.37 \\
\hline P for trend & & & \\
\hline
\end{tabular}

*Risk factors: smoking (cigarettes per day, past, current, never smokers), alcohol consumption $(0,>0-15$ $\geqslant 15$ units weekly), weekly hours of exercise, cholesterol concentration ( $\mathrm{mmol} / \mathrm{l})$, diastolic blood pressure $(\mathrm{mm} \mathrm{Hg})$, body mass index $\left(\mathrm{kg} / \mathrm{m}^{2}\right)$, percentage of forced expiratory volume in one second. Participants with missing values excluded.

Excluding participants who had angina at first screening and those not screened twice.

tExcluding participants who had ischaemia at first screening and those not screened twice.

No relation is apparent between stress and hospital admission for coronary heart disease. Higher stress is weakly associated with lower risk of ischaemia as detected by electrocardiography, admission to hospital for myocardial infarction, and mortality.

A proportion of individuals with coronary heart disease may never be treated in hospital and may have a normal resting electrocardiogram. However it seems unlikely that genuine coronary heart disease would not be associated with an increased risk of mortality in a middle aged male population followed up for over 20 years. The alternative, and more likely, explanation is that our "positive" findings were an artefact of reporting bias. Individuals who perceived and reported their lives as most stressful also tended to perceive and report more symptoms attributable to cardiovascular disease, leading to an association between heightened stress and angina. These individuals were also more likely to attend a health facility and to report their symptoms to a doctor. The mainstay of diagnosis is a history of symptoms. ${ }^{18}$ Self reported symptoms are also an important influence on decisions about hospital admission, particularly in situations where the condition is one where admission would normally be considered discretionary rather than mandatory. This is the most likely explanation for the positive association between stress and many categories of hospital admissions.

The broad categories "cardiovascular disease" and "coronary heart disease" are made up of different constituent diagnoses. Admissions for coronary heart disease did not increase with increasing stress, whereas admissions for cardiovascular disease did. We have broken down these categories to explore this discrepancy. Admissions for "coronary heart disease" are dominated by those for acute myocardial infarction, a condition where admission would normally be considered mandatory and where diagnostic classification depends primarily on objective criteria (such as the measurement of cardiac enzymes). Reporting bias will not influence these, hence admissions for myocardial infarction are not related to stress. For most classes of hospital admission the decision to admit and the classification of cause of admission depends on a combination of symptoms and signs. Where symptoms have the dominant influence, reporting bias may exert an important influence. This probably explains the weakly positive associations between angina admissions and stress and the more strongly positive association between stress and the group of admissions for cardiovascular conditions where admission was likely to be, to a substantial degree, discretionary or when diagnostic classification reflected non-specific symptoms or signs. Because of this, admissions for "cardiovascular disease" overall were positively associated with stress. Some studies have used disease diagnosed by a doctor as an "objective" outcome. ${ }^{19}$ These results suggest that it may also be influenced by reporting bias.

We are not questioning the reality of symptoms to the person experiencing them. Nor, given the strong association between angina and mortality, are we suggesting that self reported angina is, in general, a poor predictor of coronary heart disease. However, reporting tendency as well as physical disease can lead to angina being experienced and reported. This has important implications for treatment and prevention.

The weak inverse association between higher stress, ischaemia detected by electrocardiography, acute myocardial infarction, and mortality is also likely to be noncausal. In this population, socially advantaged men perceived themselves to be most stressed, leading to a confounded association between higher stress and better health. Adjustment for current occupational class attenuated but did not abolish this relation. Adjustment for other markers of social position had little additional effect (data not shown). All measures of social position are relatively crude, and statistical adjustment for them is likely to leave a residually confounded association between any socially patterned exposure and health.

In the systematic review concluding that evidence supported a causal relation between stress and cardiovascular health, these associations were most consistently observed between stress and substantially subjective end points such as self reported symptoms. ${ }^{20-23}$ It seems likely that at least some of these relations are artefactual in the same way as the ones we report here. Only one study in this review reported an association between perceived stress and mortality, in a population where stress was associated with social disadvantage and therefore likely to show a confounded association with health. ${ }^{24}$

Associations between "psychosocial" factors and objectively poorer health have been shown in other studies. ${ }^{325}{ }^{26}$ In all of these, where the social distribution of the psychosocial factor was described, "adverse" exposure was associated with social disadvantage. It is possible that these relations were also residually confounded.

In our study, heightened stress showed typical associations with unhealthy behaviour. The expected relation between stress and admissions for psychiatric disorders and experience in other studies increases our confidence in the validity of our measure of stress. ${ }^{911-13}$ However, we emphasise that our results are presented to show the way in which reporting bias and confounding can generate non-causal associations between psychosocial measures and health outcomes. These points do not depend on the validity or comprehensiveness of our stress measure. Given the predictable associations observed between conven- 
tional risk factors and a range of disease end points we have no suspicion that our results reflected an unusual study population. Furthermore, we do not believe that the issues we have highlighted have any less relevance to studies incorporating more recent or more elaborate measures of perceived stress or other self reported psychosocial constructs.

Given the plausibility of an effect of psychosocial adversity on physical health and the current popularity of psychosocial explanations for patterns of health in developed countries, it is important to clarify these issues. Spurious associations between exposures and outcomes are to be expected when both are substantially subjective. Adjustment for a measure of reporting tendency is unlikely to abolish this effect because reporting tendency is impossible to measure precisely. ${ }^{27}$ Relations with objective outcomes are more suggestive of important effects. However unless they are shown in populations where heightened exposure is not associated with social disadvantage, residual confounding is impossible to discount. Experimental studies could resolve this issue and indicate the potential, if any, for interventions targeting psychosocial exposure to improve population health.

We thank Victor Hawthorne, Charles Gillis, David Hole, and Pauline MacKinnon whose work provided us with the data required for this analysis.

Table 4 Rate ratios (95\% confidence intervals) for hospital admissions over 21 years from first screening by level of perceived stress at first screening

\begin{tabular}{|c|c|c|c|c|}
\hline Perceived stress & No of admissions & Adjusted for age & $\begin{array}{l}\text { Adjusted for age and social } \\
\text { class }\end{array}$ & $\begin{array}{l}\text { Adjusted for age, social class, and } \\
\text { risk factors* }\end{array}$ \\
\hline \multicolumn{5}{|c|}{ All cause, general hospital: } \\
\hline High & 1913 & $1.10(0.98$ to 1.23$)$ & 1.15 (1.03 to 1.29$)$ & 1.13 (1.01 to 1.27$)$ \\
\hline Medium & 6749 & 0.97 (0.89 to 1.05$)$ & 1.04 (0.96 to 1.13$)$ & $1.03(0.95$ to 1.11$)$ \\
\hline Low & 4240 & 1.00 & 1.00 & 1.00 \\
\hline $\mathrm{P}$ for trend & & 0.32 & 0.021 & 0.048 \\
\hline \multicolumn{5}{|c|}{ Cardiovascular disease: } \\
\hline High & 524 & 1.14 (0.94 to 1.37$)$ & 1.22 (1.01 to 1.47$)$ & 1.20 (1.00 to 1.45$)$ \\
\hline Medium & 1740 & 0.96 (0.84 to 1.09$)$ & 1.06 (0.93 to 1.21$)$ & 1.03 (0.91 to 1.18$)$ \\
\hline Low & 1105 & 1.00 & 1.00 & 1.00 \\
\hline $\mathrm{P}$ for trend & & 0.38 & 0.049 & 0.081 \\
\hline \multicolumn{5}{|c|}{ Coronary heart disease: } \\
\hline High & 215 & $0.94(0.72$ to 1.24$)$ & $1.00(0.76$ to 1.33$)$ & $1.00(0.76$ to 1.32$)$ \\
\hline Medium & 845 & 0.94 (0.78 to 1.14$)$ & 1.02 (0.84 to 1.23$)$ & 0.96 (0.79 to 1.16$)$ \\
\hline Low & 544 & 1.00 & 1.00 & 1.00 \\
\hline $\mathrm{P}$ for trend & & 0.57 & 0.93 & 0.90 \\
\hline \multicolumn{5}{|c|}{ Acute myocardial infarction: } \\
\hline High & 91 & 0.79 (0.59 to 1.06$)$ & $0.82(0.61$ to 1.10$)$ & $0.86(0.65$ to 1.15$)$ \\
\hline Medium & 399 & 0.89 (0.74 to 1.08$)$ & $0.93(0.77$ to 1.14$)$ & $0.93(0.76$ to 1.13$)$ \\
\hline Low & 272 & 1.00 & 1.00 & 1.00 \\
\hline $\mathrm{P}$ for trend & & 0.10 & 0.20 & 0.29 \\
\hline \multicolumn{5}{|l|}{ Angina: } \\
\hline High & 45 & $1.17(0.69$ to 2.00$)$ & 1.35 (0.79 to 2.31$)$ & $1.32(0.78$ to 2.26$)$ \\
\hline Medium & 135 & 0.87 (0.60 to 1.27$)$ & 1.01 (0.69 to 1.49$)$ & $1.01(0.68$ to 1.50$)$ \\
\hline Low & 94 & 1.00 & 1.00 & 1.00 \\
\hline$P$ for trend & & 0.80 & 0.35 & 0.38 \\
\hline \multicolumn{5}{|c|}{ Hypertension, varicose veins, and ill defined conditions†: } \\
\hline High & 107 & 1.72 (1.25 to 2.37$)$ & 1.83 (1.32 to 2.53$)$ & 1.91 (1.38 to 2.64$)$ \\
\hline Medium & 322 & 1.26 (1.00 to 1.61$)$ & 1.36 (1.06 to 1.73$)$ & 1.39 (1.09 to 1.77$)$ \\
\hline Low & 153 & 1.00 & 1.00 & 1.00 \\
\hline $\mathrm{P}$ for trend & & 0.001 & $<0.001$ & $<0.001$ \\
\hline \multicolumn{5}{|c|}{ Other cardiovascular disease $\ddagger$ : } \\
\hline High & 253 & $1.08(0.84$ to 1.40$)$ & $1.14(0.88$ to 1.48$)$ & $1.14(0.88$ to 1.48$)$ \\
\hline Medium & 809 & 0.88 (0.74 to 1.06$)$ & 0.98 (0.81 to 1.17$)$ & 0.99 (0.82 to 1.19$)$ \\
\hline Low & 550 & 1.00 & 1.00 & 1.00 \\
\hline$P$ for trend & & 0.96 & 0.45 & 0.43 \\
\hline \multicolumn{5}{|c|}{ Psychiatric disorders: } \\
\hline High & 103 & 2.20 (1.33 to 3.65$)$ & 2.34 (1.41 to 3.91$)$ & $\S$ \\
\hline Medium & 212 & 1.10 (0.76 to 1.60$)$ & 1.18 (0.80 to 1.73$)$ & $\S$ \\
\hline Low & 114 & 1.00 & 1.00 & $\S$ \\
\hline$P$ for trend & & 0.004 & 0.002 & \\
\hline
\end{tabular}

Participants in each stress category: high stress $n=743$, medium stress $n=3037$, and low stress $n=1826$.

*Risk factors: smoking (cigarettes per day, past, current, never smokers), alcohol consumption $(0,>0-15, \geqslant 15$ units weekly), weekly hours of exercise, cholesterol concentration $(\mathrm{mmol} / \mathrm{l})$, diastolic blood pressure $(\mathrm{mm} \mathrm{Hg})$, body mass index $\left(\mathrm{kg} / \mathrm{m}^{2}\right)$, percentage of forced expiratory volume in one second. Participants with missing values excluded.

tIncludes ICD9 codes 401 (hypertension) 189 admissions; 429 (ill defined heart disease) 12 admissions; 454 (lower limb varicose veins) 156 admissions; 455 (haemorrhoids) 147 admissions; 456 (other varicose veins) 35 admissions; 457 (lympho-oedema) 2 admissions; 458 (hypotension) 14 admissions; 459 (othe circulatory disorders) 27 admissions

fIncludes ICD9 codes 393-398 (chronic rheumatic heart disease) 43 admissions; $402-405$ (hypertensive disease other than essential hypertension) 15 admissions: 415-417 (diseases of the pulmonary circulation) 67 admissions; 420-425 (diseases of the pericardium, endocardium, and myocardium) 73 admissions; $426-428$ (conduction disorders, dysrhythmias, and heart failure) 469 admissions; 430-438 (cerebrovascular disease) 401 admissions; $440-448$ (diseases of arteries, arterioles, and capillaries) 449 admissions; 451 (phlebitis) 60 admissions; 453 (venous embolism and thrombosis) 35 admissions.

$\S$ Not reported as cardiovascular risk factors were not expected to confound a relation with psychiatric disease. 
Table 5 Hazard ratios (95\% confidence intervals) for mortality over 21 years from first screening by level of perceived stress at first screening

Perceived stress*

Adjusted for age

Adjusted for age and social class

Adjusted for age, social class, and

All cause (1601 deaths): risk factors $†$

\begin{tabular}{lccc}
\hline High & $0.90(0.77$ to 1.05$)$ & $0.96(0.82$ to 1.11$)$ & $0.94(0.81$ to 1.11$)$ \\
\hline Medium & $0.85(0.76$ to 0.94$)$ & $0.93(0.83$ to 1.04$)$ & $0.92(0.82$ to 1.03$)$ \\
\hline Low & 1.00 & 1.00 & 1.00 \\
\hline P for trend & 0.030 & 0.40 & 0.30 \\
\hline Cardiovascular disease (820 deaths): & & & \\
\hline High & $0.83(0.72$ to 0.97$)$ & $0.91(0.78$ to 1.06$)$ & $0.91(0.78$ to 1.06$)$ \\
\hline Medium & $0.84(0.68$ to 1.05$)$ & $0.90(0.72$ to 1.13$)$ & $0.91(0.73$ to 1.14$)$ \\
\hline Low & 1.00 & 1.00 & 1.00 \\
\hline P for trend & 0.036 & 0.25 & 0.28 \\
\hline Coronary heart disease (609 death): & & & $0.95(0.74$ to 1.23$)$ \\
\hline High & $0.89(0.69$ to 1.15$)$ & $0.96(0.80$ to 1.14$)$ & $0.95(0.81$ to 1.16$)$ \\
\hline Medium & $0.87(0.73$ to 1.04$)$ & 1.00 & 1.00 \\
\hline Low & 1.00 & 0.64 & 0.79 \\
\hline P for trend & 0.20 & &
\end{tabular}

*High, $\mathrm{n}=743$; medium, $\mathrm{n}=3037$; low, $\mathrm{n}=1826$.

tRisk factors: smoking (cigarettes per day, past, current, or never smokers), alcohol consumption $(0,>0-15, \geqslant 15$ units weekly), weekly hours of exercise, cholesterol concentration $(\mathrm{mmol} / \mathrm{l})$, diastolic blood pressure $(\mathrm{mm} \mathrm{Hg})$, body mass index $\left(\mathrm{kg} / \mathrm{m}^{2}\right)$, percentage of forced expiratory volume in one second. Participants with missing values excluded.

\section{What is already known on this topic}

Higher psychological stress has predicted coronary heart disease in several observational studies

Exposure to stress and heart disease outcomes were often based on self report so that a general tendency to negative perceptions may have generated a spurious association between higher perceived stress and heart disease symptoms

\section{What this study adds}

Perceived stress was strongly related to subjective symptoms of heart disease, including those leading to hospital admission

However, stress showed a weakly inverse relation to all objective indices of heart disease: socially advantaged men perceived themselves to be most stressed, and the "protective" effect of stress was probably attributable to residual confounding

Suggestions that psychological stress is an important determinant of heart disease may be premature

Contributors: GDS conceived the study and will act as guarantor for the paper. GDS, JM, and CH obtained funding. JM and CM undertook the analyses; JM wrote the first draft of the paper and was responsible for subsequent revisions in the light of critical comments from all authors.

Funding: This work was supported by a grant within phase two of the health variations research programme of the Economic and Social Research Council. Preliminary analyses on this dataset were undertaken by JM while he was supported by a clinical epidemiology training fellowship from the Wellcome Trust.

Competing interests: None declared.

1 Davison C, Davey Smith G, Frankel SJ. Lay epidemiology and the prevention paradox: the implications of the coronary candidate for health promotion. Sociol Health Illness 1991;13:1-19.

2 Greenwood DC, Muir KR, Packham CJ, Madeley RJ. Coronary heart disease: a review of the role of psychosocial stress and social support. J Pub Health Med 1996;18:221-31.
3 Hemingway H, Marmot M. Evidence based cardiology: psychosocial factors in the aetiology and prognosis of coronary heart disease: systematic review of prospective cohort studies BMJ 1999;318:1460-7.

4 Brunner E. Stress and the biology of inequality. BMJ 1997;314:1472-6.

5 Schachter S, Silverstein B, Kozlowski LT, Herman CP, Liebling B. Effects of stress on cigarette smoking and urinary pH. J Exp Psychol: Gen 1977;106:24-30

6 Watson D, Clark LA. Negative affectivity: the disposition to experience aversive emotional states. Psychol Bull 1984;96:465-90.

7 Davey Smith G, Harding S. Is control at work the key to socio-economic gradients in mortality? Lancet 1997;350:1369-70.

8 Wainwright D, Calnan M. Rethinking the work stress "epidemic." Eur J Public Health 2000;10:231-4.

9 Heslop P, Davey Smith G, Carroll D, Macleod J, Hart C. Perceived stress and coronary heart disease risk-factors: the contribution of socioeconomic position. Br J Health Psychol 2001;6:167-78.

10 Rose GA, Blackburn H, Gillum RF, Prineas RJ. Cardiovascular survey methods. Geneva: World Health Organization, 1982.

11 Chapman JM, Reeder LG, Massey J, Borun ER, Picken B, Browning GG, et al. Relationships of stress, tranquilizers and plasma cholesterol levels in a sample population under study for coronary heart disease. Am J Epidemiol 1966;83:537-47.

12 Reeder LG, Schrama PGM, Dirken JM. Stress and cardiovascular health: an international cooperative study: I. Soc Sci Med 1973;7:573-84.

13 Haynes SG, Levine S, Scotch N, Feinleib M, Kannel WB. The relationship of psychosocial factors to coronary heart disease in the Framingham Study: methods and risk-factors. Am J Epidemiol 1978;107:362-83.

14 Reeder LG, Chapman JM, Coulson AH. Socioenvironmental stress, tranquilizers and cardiovascular disease. Proceedings of the Excerpta Medica International Congress Series, 1968;182:226-38.

15 Davey Smith G, Hart C, Hole D, MacKinnon P, Gillis C, Watt G, et al. Education and occupational social class: which is the more important indicator of mortality risk? J Epidemiol Community Health 1998;52:153-60.

16 Kendrick S, Clarke J. The Scottish record linkage system. Health Bull 1993;51:72-9.

17 Glynn RJ, Buring JE. Ways of measuring rates of recurrent events. BMJ 1996;312:364-6.

18 Sackett DL. Clinical diagnosis and the clinical laboratory. Clin Invest Med 1978;1:37-43

19 Marmot MG, Bosma H, Hemingway H, Brunner E, Stansfeld S. Contribution of job control and other risk-factors to social variations in coronary heart disease incidence. Lancet 1997;350:235-9.

20 Haynes SG, Feinleib M, Levine S, Scotch N, Kannel WB. The relationship of psychosocial factors to coronary heart disease in the Framingham study. II Prevalence of coronary heart disease. Am J Epidemiol 1978;107:384-402

21 Haynes SG, Feinleib M, Kannel WB. The relationship of psychosocial factors to coronary heart disease in the Framingham study. III. Eight-year incidence of coronary heart disease. Am J Epidemiol 1980:111:37-58.

22 Hagman M, Wilhelmsen L, Wedel H, Pennert K. Risk-factors for angina pectoris in a population study of Swedish men. J Chron Dis 1987;40: 265-75

23 Hollis JF, Connett JE, Stevens VJ, Greenlick MR. Stressful life events, type A behaviour, and the prediction of cardiovascular and total mortality over six years. J Behav Med 1990;13:263-80.

24 Rosengren A, Tibblin G, Wilhelmsen L. Self-perceived psychological stress and incidence of coronary artery disease in middle-aged men. Am J Cardiol 1991;68:1171-5.

25 Orth-Gomer K, Wamala SP, Horsten M, Schenck-Gustafsson K, Schneiderman N, Mittleman MA. Marital stress worsens prognosis in women with coronary heart disease. The Stockholm Female Coronary Risk Study. JAMA 2000;284:3008-14. 
26 Haines A, Cooper J, Meade TW. Psychological characteristics and fatal ischaemic heart disease. Heart 2001;85:385-9.

27 Macleod J, Davey Smith G, Heslop P, Metcalfe C, Carroll D. Limitations of attempts to adjust for reporting tendency in observational studies of stress and self-reported coronary heart disease. J Epidemiol Community Health 2002;56:76-7.

(Accepted 29 November 2001)

\section{Commentary: Psychosocial factors and health-strengthening the evidence base}

John Lynch

Reading the paper by Macleod and colleagues I am reminded of growing up in Australia, where my parents impressed upon me the importance of not being a "whinger." In fact, it was common for my grandmother when asked how she was feeling to reply "musn't grumble." Such stoicism even in the face of malaise was thought to be a positive personality disposition.

In an ingenious use of data collected in the early 1970 s, Macleod and colleagues utilised baseline and follow up self reported and clinical data, combined with subsequent hospital admissions and mortality to show that men who reported themselves as feeling more tense, nervous, and exhausted by the stress of daily activities were more likely to have angina (derived from self report) and to be admitted to hospital for certain conditions (influenced by self reports to the attending physician). However, higher stress was not associated with hospital admissions, for which there were more objective criteria, such as myocardial infarction. Nor was stress associated with mortality. The authors concluded that their results showed a reporting bias-that is, people who viewed their lives as more stressful were also more likely to report more symptoms attributable to cardiovascular disease.

This study will evoke a range of responses. Some may point out that the stress measure used here is rather old fashioned and so the results are not widely applicable. Contemporary psychosocial research investigates constructs like hostility, hopelessness, and depression, which are defined more precisely than stress, measured using more refined instruments, and have been linked to objective outcomes. ${ }^{12}$ Others will argue that this is a good example of how the effects of psychosocial exposures on health may have been oversold. The nature of the effects of self reported stress found here are reasonably typical of the evidence generated from studies linking job stress and self reported outcomes like angina, and this paper may aid in greater understanding of such links. ${ }^{3}$

This study represents a cogent empirical example of how reporting bias can generate associations between self reported exposures and outcomes. This does not necessarily mean that all such associations are spurious, but it does illustrate the potential for report- ing bias. Furthermore, Macleod and colleagues argued that when a psychosocial exposure is not linked to social disadvantage, associations between self reported psychosocial stress and self reported outcomes may be the result of reporting bias. When the psychosocial exposure is patterned by socioeconomic disadvantage associations may be-depending on the outcome- the result of reporting bias or residual confounding by unmeasured socioeconomic factors from across the life course. ${ }^{4}$ These points are well defined challenges for those of us trying to gain greater understanding of the role of psychosocial exposures. Investigators now have more reason than ever to address reporting bias and residual confounding in investigating the health effects of psychosocial exposures. They imply that the most convincing evidence for a causal role of psychosocial exposures will come from studies that have objective and self reported outcomes and have measured all the relevant covariates including other psychosocial factors and socioeconomic factors from all stages of the life course. ${ }^{5} \mathrm{I}$ think that for many extant studies that will be tough to achieve. But as I have an aversion to whingeing perhaps it's just better to get on and try to ensure that future studies of the health effects of psychosocial exposures can address these challenges. Macleod and colleagues' results should not be seen as a threat to the study of psychosocial exposures and health. On the contrary, they help to strengthen the discipline.

Competing interests: None declared.

1 Everson SA, Goldberg DE, Kaplan GA, Cohen RD, Pukkala E, Tuomilento J, et al. Hopelessness and risk of mortality and incidence of myocardial infarction and cancer. Psychosom Med 1996;58:113-21.]

2 Iribarren C, Sidney S, Bild DE, Liu K, Markovitz JW, Roseman JM, et al. Association of hostility with coronary artery calcification in young adults-the CARDIA study. JAMA 2000;283:2546-51

3 Bosma H, Marmot MG, Hemingway H, Nicholson AC, Brunner E, Stansfeld SA. Low job control and risk of coronary heart disease in Whitehal II (prospective cohort) study BMJ 1997;314:558-65.

4 J Macleod J, Davey Smith G, Heslop P, Metcalfe C, Carroll D, Hart C. Are the effects of psychosocial exposures attributable to confounding? Evidence from a prospective observational study on psychological stress and mortality. J Epidemiol Community Health 2001;5:878-84.

5 Harper S, Lynch JW, Everson SA, Hsu W-L, Raghunathan T, Kaplan GA Lifecourse socioeconomic position and depression, cynical hostility and hopelessness in adulthood. Int J Epidemiol 2002. (In press.)
Department of Epidemiology, School of Public Health, University of Michigan, Ann Arbor, MI 48109-2029, USA jwlynch@umich.edu 